\title{
Sensors for the Determination of Organic Load (Chemical Oxygen Demand) Utilizing Copper/Copper Oxide Nanoparticle Electrodes
}

\section{S \\ DE SENSORS I BIOSENSORS}

Abstract

This research project develops electronic sensors for determination of Chemical Oxygen Demand (COD) with the technique of cyclic voltammetry. This detection is based on the changes of current intensity followed by the oxidation reaction of organic substances in wastewater, which takes place on the surface of electrodes. Copper electrodes are chosen as the working electrodes based on the fact that copper in alkaline media acts as a powerful electrocatalyst for oxidation of aminoacids and carbohydrates, which are believed to be the major culprits for organic pollution. In this work, three kinds of copper/copper oxide electrodes were studied: electrodeposited copper nanoparticle electrode, copper nanoparticle-graphite composite electrode and copper oxide nanoparticle-graphite composite electrode.
Qing Wang, Manel del Valle

Group of Sensors and Biosensors, Department of Chemistry, Universitat Autònoma de Barcelona, 08193 Bellaterra, Barcelona, Spain

E-mail: qing.wang2@e-campus.uab.cat manel.delvalle@uab.es

\section{Chemical Oxygen Demand (COD)}

Free oxygen dissolved in water is very important for supporting aquatic life in ecosystems. The decomposition of organic contaminants will consume oxygen, which will affect the survival and qualities of these ecosystems. Chemical Oxygen Demand (COD) is a widely used parameter in analysing the extent of organic contamination of water samples[1]. The conventional method for COD determination is defined as the number of oxygen equivalents consumed in the oxidation of organic compounds by strong oxidizing agents such as dichromate and permanganate. However, this method requires the timeconsuming process of refluxing samples to achieve more complete oxidation; reproducibility of the results are dependent on the skill of the operator and expensive and toxic reagents. Electrochemistry methods of analysis are becoming more and more attractive for COD quantitation because they could be simple, accurate, sensitive and safe[2].

\section{Why "copper"?}

Copper in alkaline media acts as a powerful electrocatalyst for oxidation of carbohydrates and aminoacids. The oxidation process will result in the changes of current intensity, which can act as the signal for detection and analysis. In this sense, three electrodes with copper incorporated in different forms are designed: surface electrodeposited $\mathrm{CuO} / \mathrm{Cu}$ electrode (E1), copper nanoparticle-graphite composite electrode (E2), copper dioxide nanoparticle-graphite composite electrode (E3) and nickel/copper alloy nanoparticle-graphite composite electrode (E4).

\section{Fabrication of Electrodes}

1. Nafion film covered eletrodeposited $\mathrm{CuO} / \mathrm{Cu}$ electrode $\mathbf{E} 1$

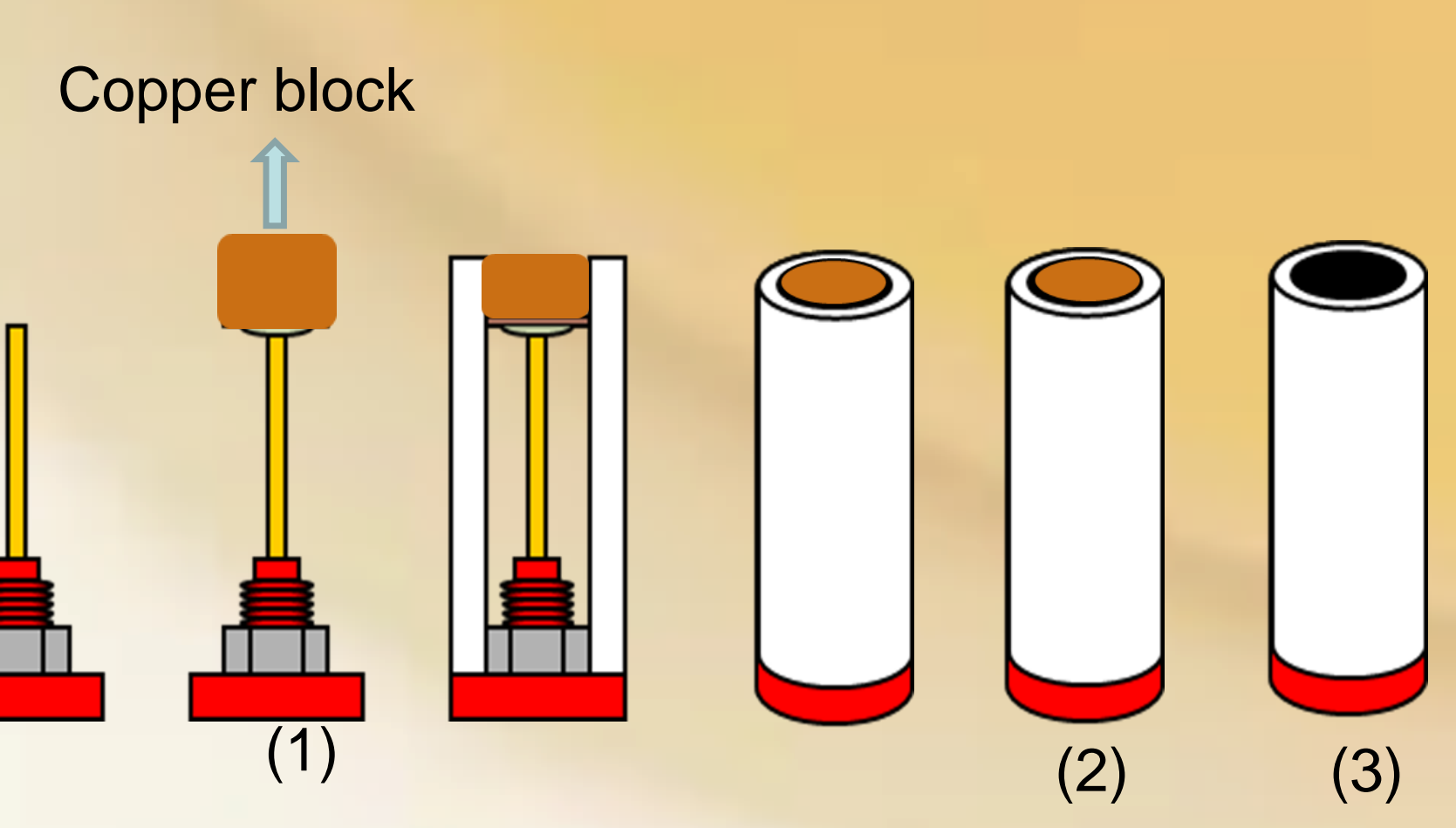

Fix the copper block to electrical connector Cover the copper surface with Nafion film Electrodeposit

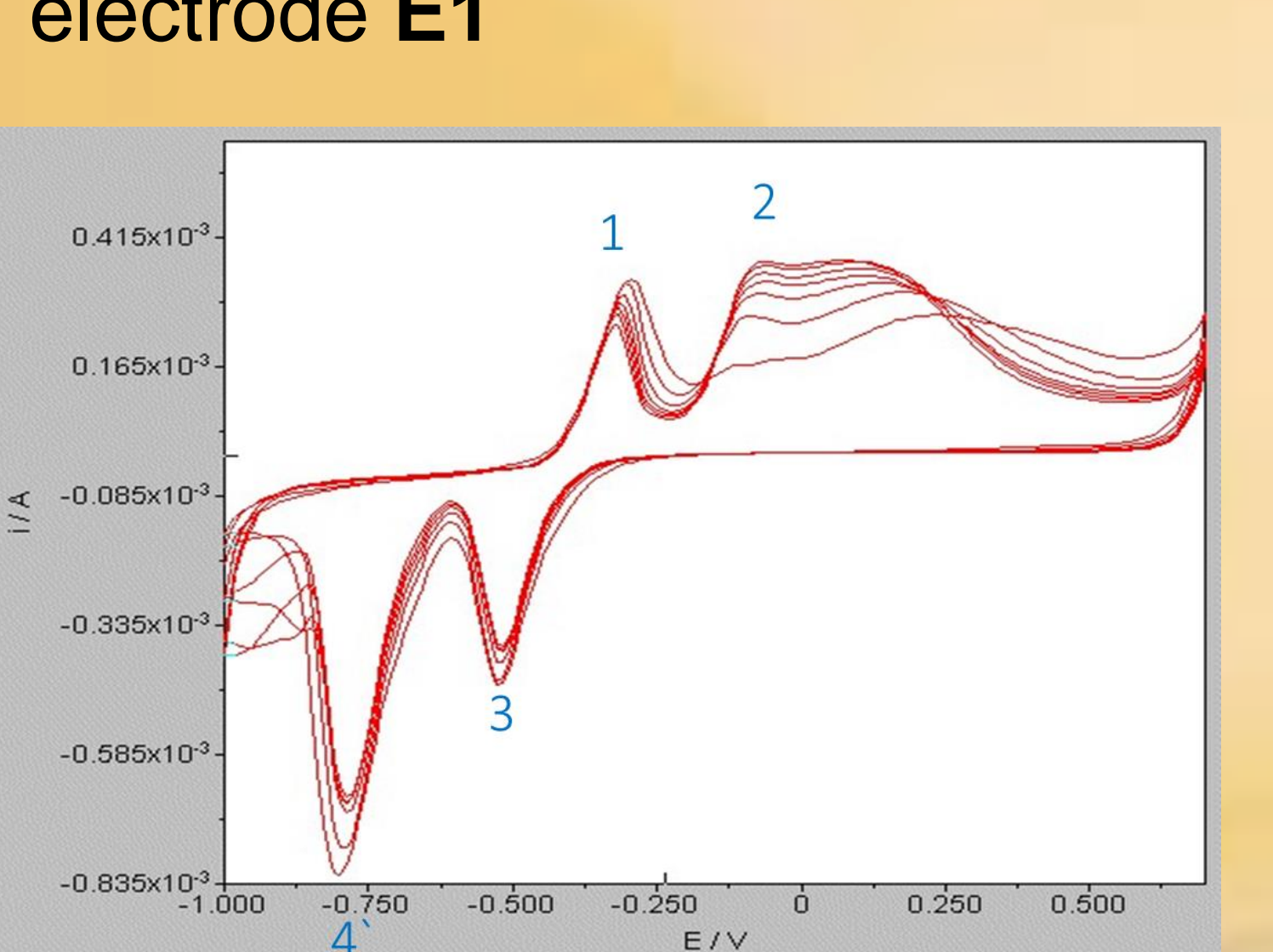
Figure 1. Series of cyclic voltammograms during preparation of the metallic
copper electrode 1 in $0.05 \mathrm{M} \mathrm{NaOH}-0.1 \mathrm{M} \mathrm{KCl}$ mixed solution. Potential scan
window -10 to Peak 1: Formation of a first layer of $\mathrm{Cu}_{2} \mathrm{O}$;

Peak 2: Formation of a second mixed layer of $\mathrm{CuO} / \mathrm{Cu}(\mathrm{OH})_{2}$ Peak 3: Reduction reaction of $\mathrm{Cu}(\mathrm{I}) / \mathrm{Cu}(\mathrm{I}$

Peak 4: Reduction reaction of $\mathrm{Cu}(\mathrm{I}) / \mathrm{Cu}(0)$;

2. Nanoparticles composite Electrodes E2, E3 and E4

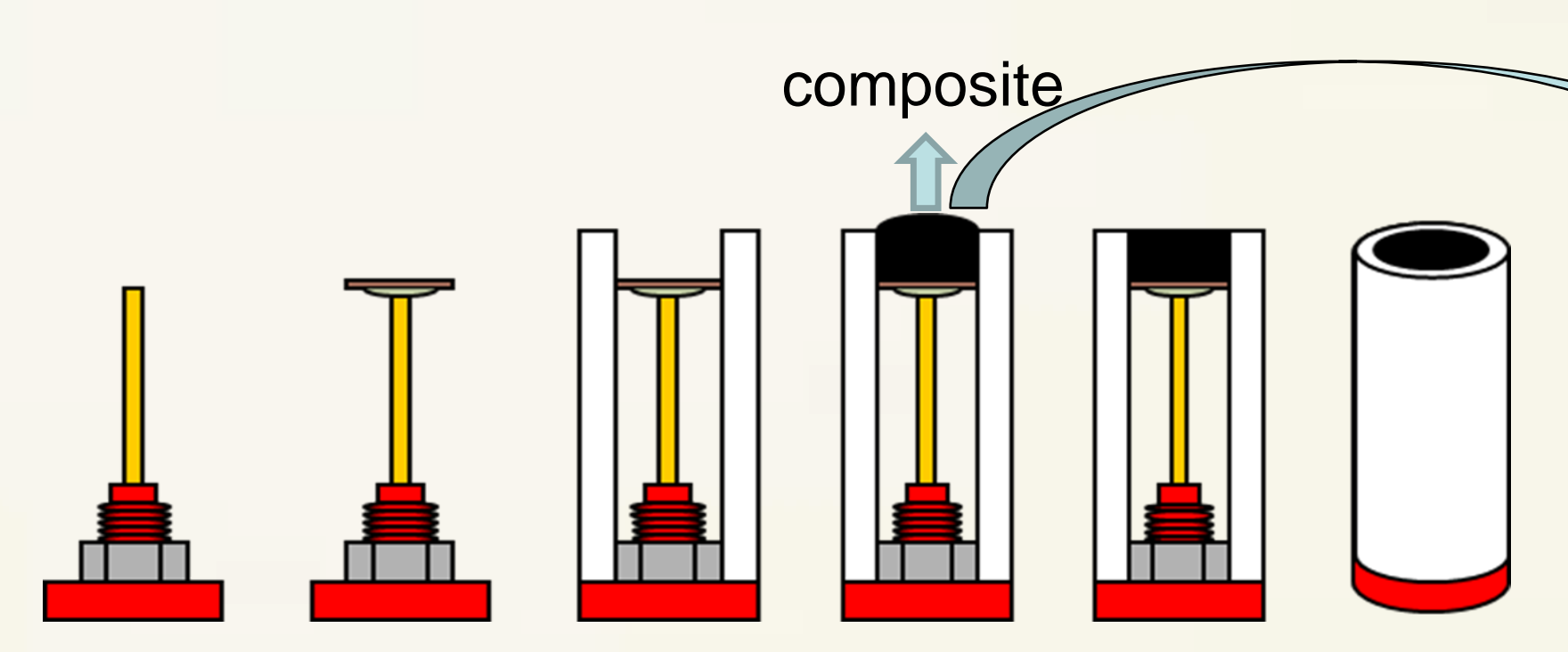

Scheme 2. Fabrication of Nanoparticle Composite Electrodes

(1) Fix the copper disk to electrical connecto

(2) Introduction of composite mixture

(3) Polishing with emery paper
E2: Cu nanoparticles + graphite + epoxy E3: CuO nanoparticles + graphite + epoxy E4: Ni/Cu alloy nanoparticles + graphite + epoxy

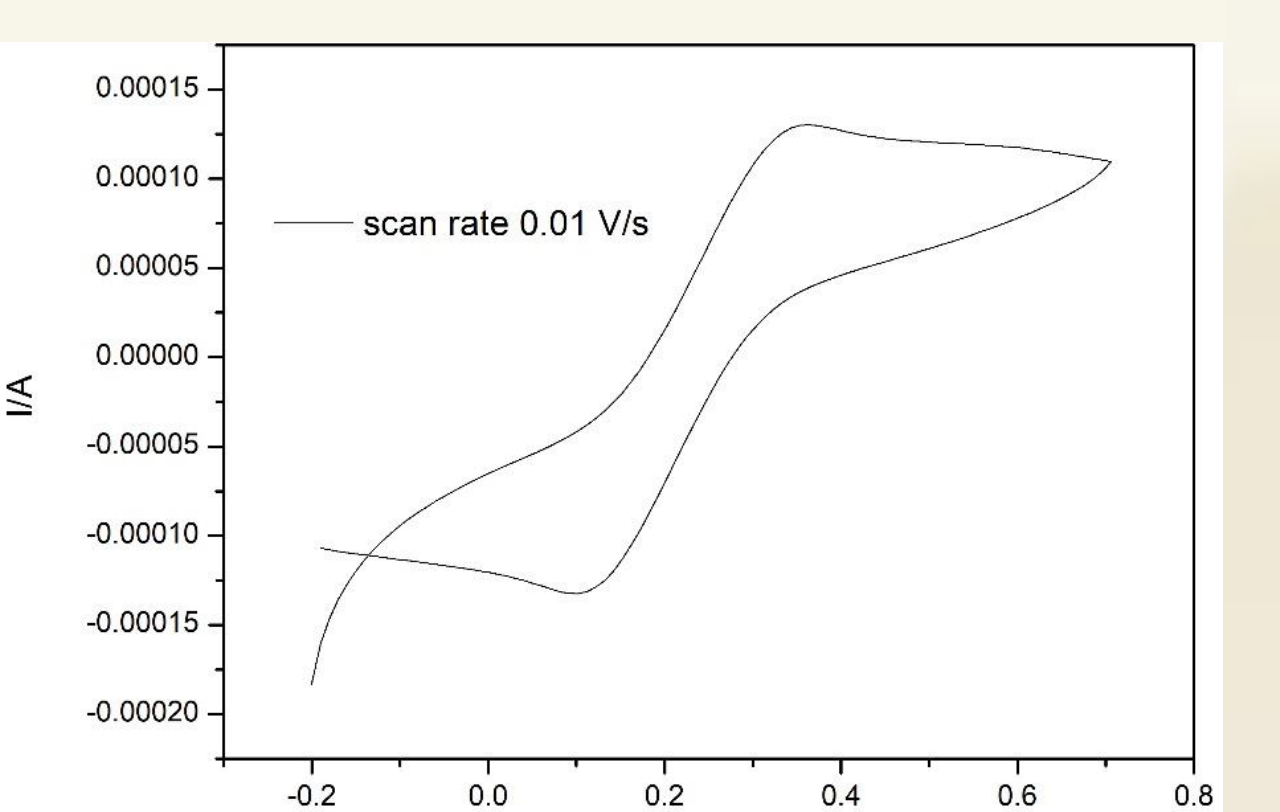

Figure 2. Cyclic voltammogram of electrode $\mathbf{E} 2$ in the solution of $0.05 \mathrm{M} \mathrm{K}_{4}\left[\mathrm{Fe}(\mathrm{CN})_{6}\right] / \mathrm{K}_{3}\left[\mathrm{Fe}(\mathrm{CN})_{6}\right]$.

\section{Application of four electrodes}
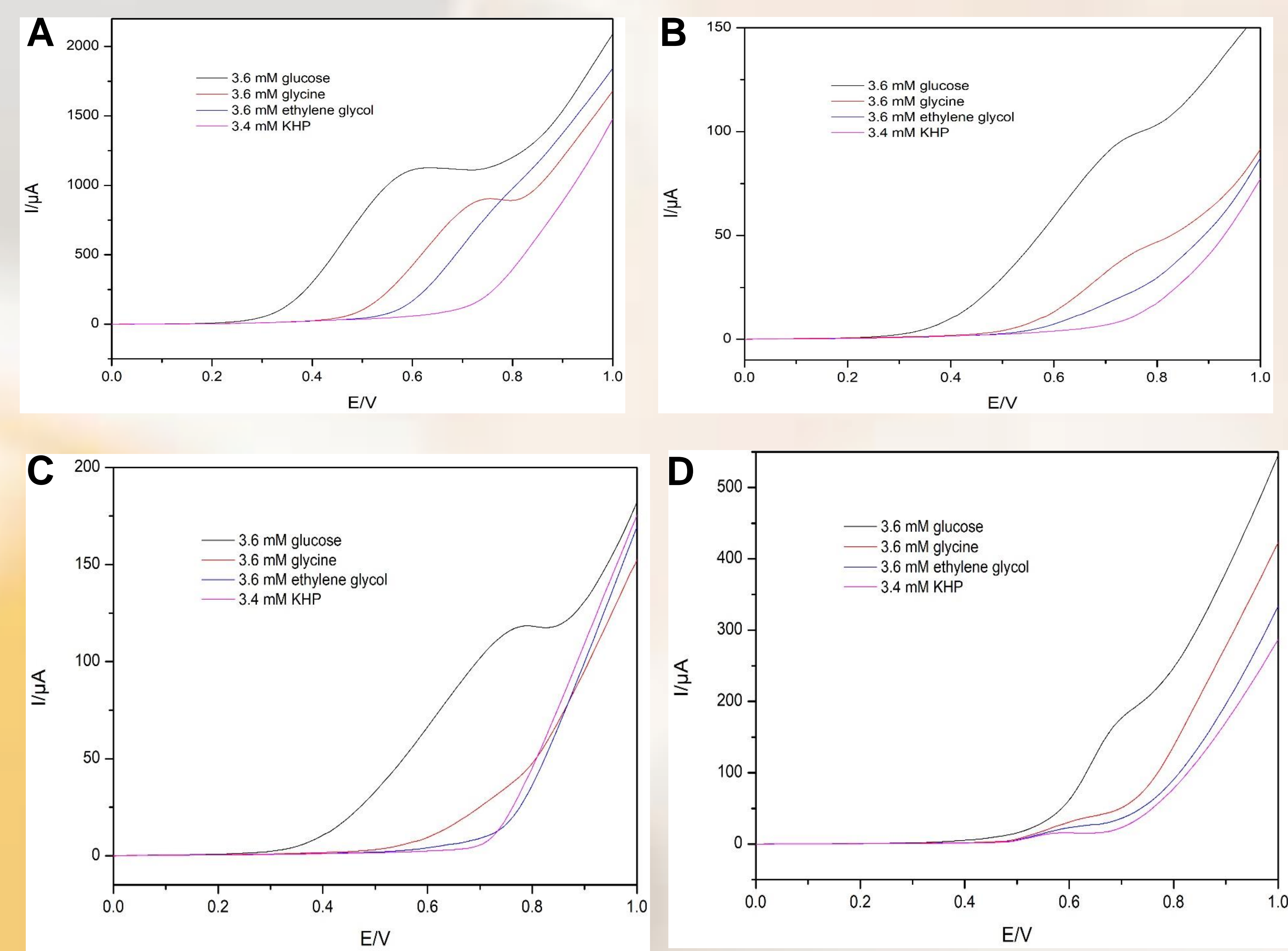

Figure 3. Oxidation curves of cyclic voltammetric responses of deposited copper electrode E1 (A), E2 (B), E3 (C) and E4 (D) to glucose, glycine, ethylene glycol, and potassium hydrogen p.
$+0.7 \mathrm{~V} \mathrm{vs.} \mathrm{Ag/AgCl}(3.0 \mathrm{M} \mathrm{KCl})$. Scan rate, $50 \mathrm{mV} / \mathrm{s}$.

\section{PCA results}

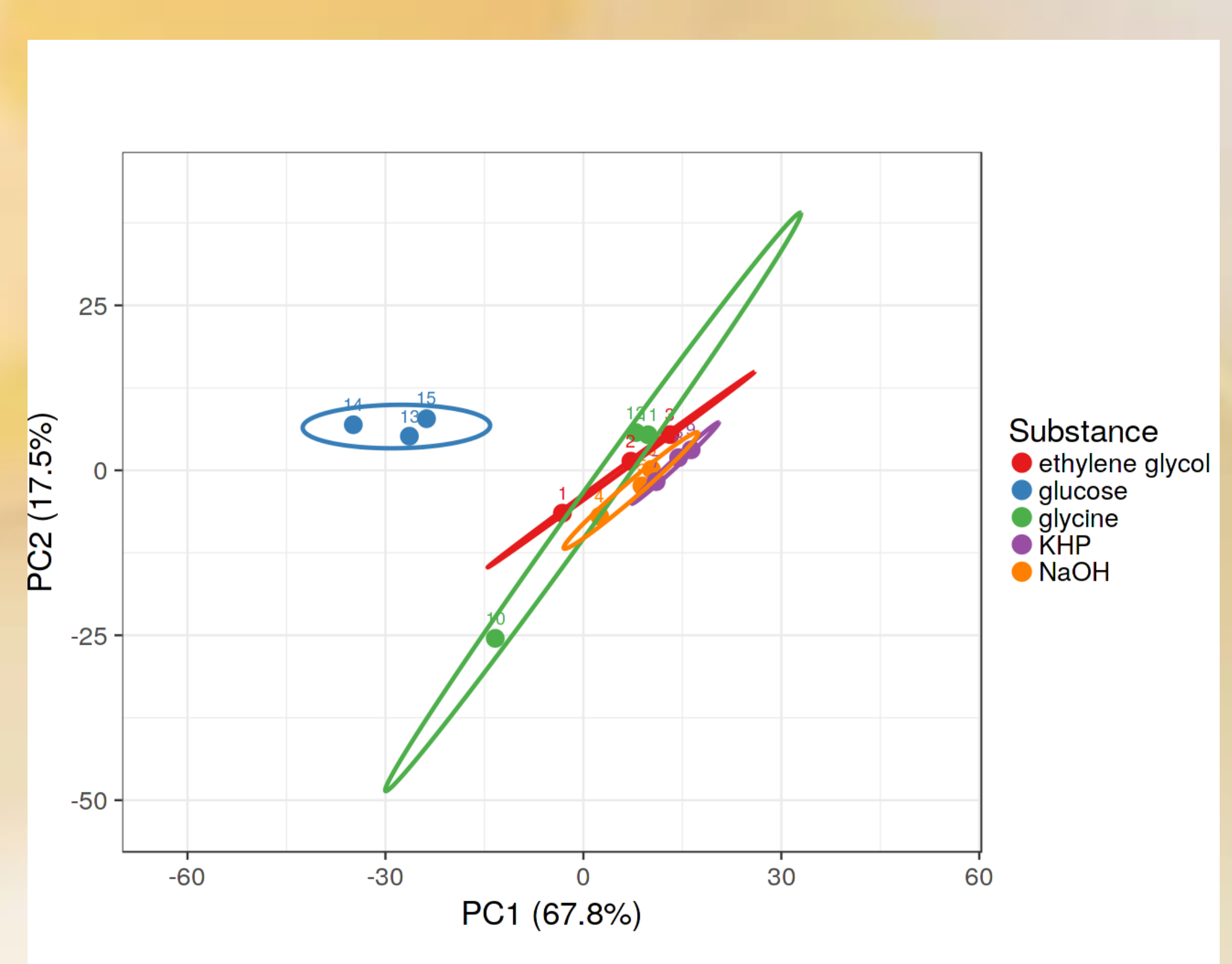
Figure 4 . Score plot of the first two components obtained after PCA
analysis.

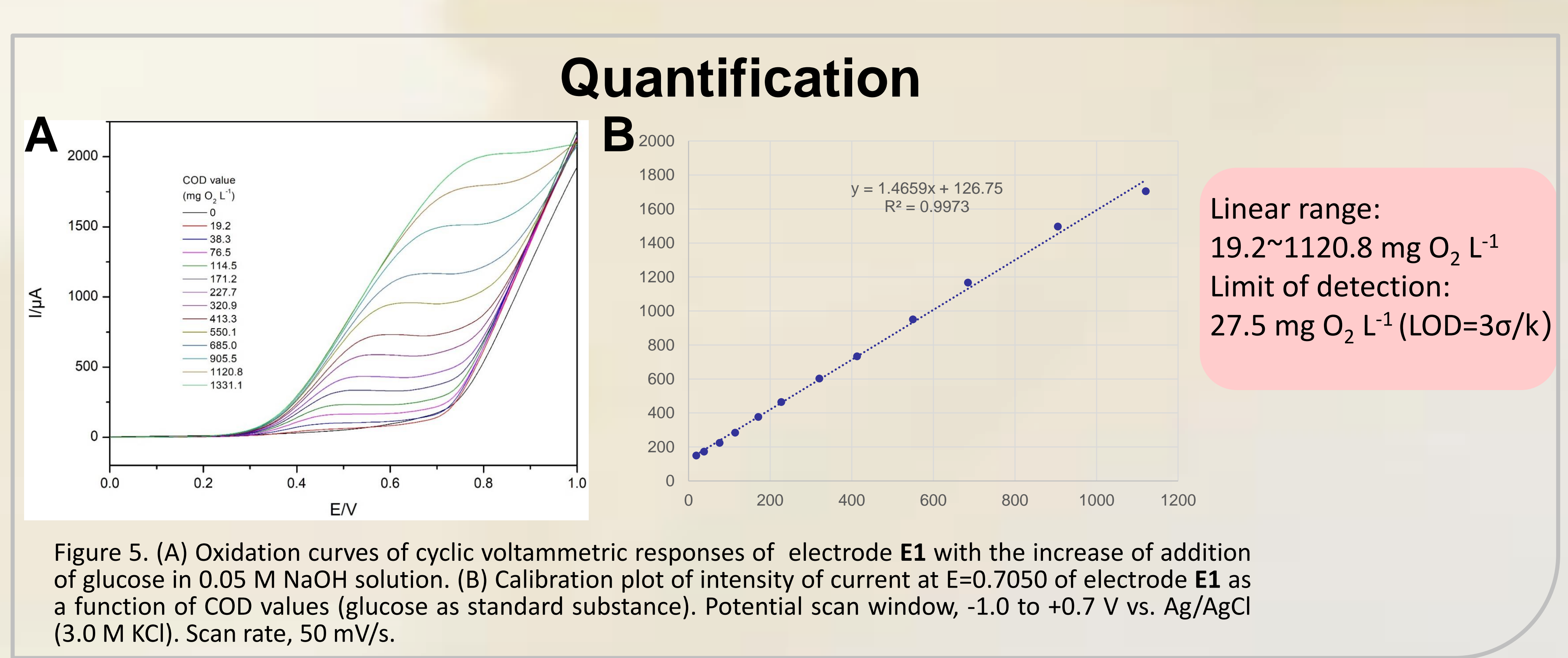

\section{Conclusions}

The performed calibrations showed that the electrodeposited copper electrode can detect COD values using standard substances. The linear range is from 19.2 to $1120.8 \mathrm{mg} \mathrm{O}_{2} \mathrm{~L}^{-1}$ and the limit of detection is $27.5 \mathrm{mg} \mathrm{O}_{2} \mathrm{~L}^{-1}$, which shows a good performance. The conducted PCA analysis implies that this sensor array can be used to distinguish different water samples that contains different organic contaminants. The ongoing work is to optimize the detection condition and to analyse real samples.

\section{Acknowledgements}

Financial support for this work was provided by Spanish Ministry of Science and Innovation trough the project CTQ 2016-80170 and by program ICREA Academia from Generalitat de Catalunya. Qing Wang thanks the support of Universitat Autonoma de Barcelona and China Scholarship Council for the UAB-CSC joint scholarship.
UAB

Universitat Autònoma de Barcelona

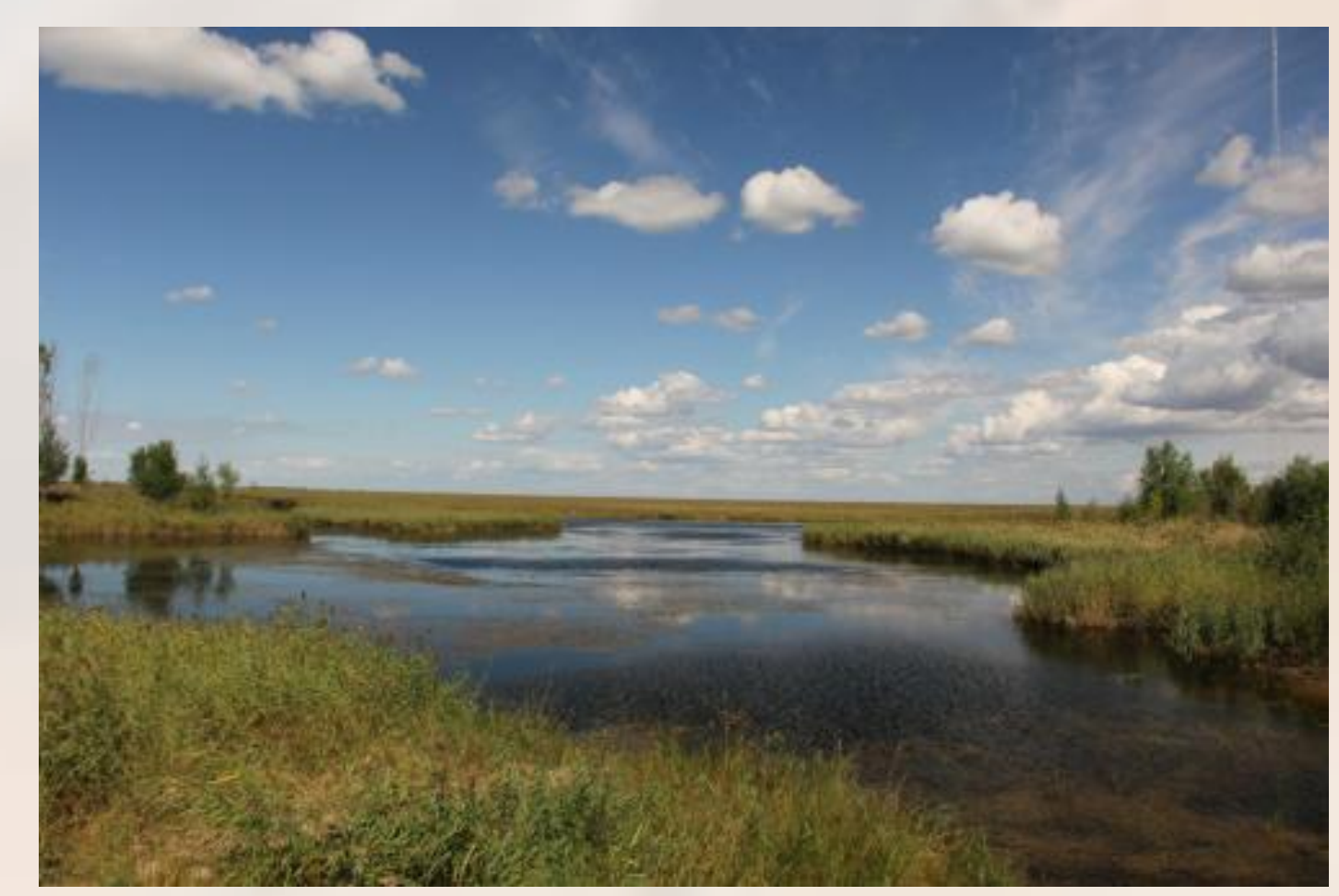

